\title{
Chemische Analyse des Meteoriten von Migheja.
}

\author{
Von \\ P. Melikofr und W. Krschischanowskr.
}

Der Meteorit von Migheja fiel am 9. Juni 1889 im Dorfe Migheja (Gouvernement Kherson, Elisabethgrader Kreis). Er bildet eine verhältnismälsig spröde, von amorpher Kohle durchdrungene Masse. Bei Berührung mit Gegenständen hinterlälst der Meteorit auf ihnen eine Spur und giebt einen schwarzen Strich auf Papier. Seine Rinde hat eine matte Oberfläche mit dunkelgrauem Schiller; im Innern der Masse sind weifse, mikroskopische Bestandteile bemerkbar. Beim Reiben verbreitet der Meteorit einen charakteristischen Geruch, an bituminöse Körper erinnernd.

Bevor dieser Meteorit in das mineralogische Kabinett der Neurussischen Universität gebracht wurde, wurde ein Teil desselben von Stanislads Mennier für das Pariser Museum erworben und der chemischen Analyse unterworfen. ${ }^{1} \mathrm{Da}$ aber die Analyse dieses Meteoriten von MENNIER nicht vollständig war, nahmen wir dieselbe vor. Der Direktor des Mineralogischen Kabinetts der Neurussischen Universität, Prof. Dr. R. A. Prender, stellte uns einen Teil derselben zur Verfügung, wofür wir ihm unseren Dank aussprechen.

Der Meteorit von Migheja enthält 1.4\% hygroskopischen Wassers, welches er bei $105^{\circ} \mathrm{C}$. verliert. Dieses Wasser ist ohne Zweifel tellurischer Herkunft, denn nach dem Austrocknen nimmt der Meteorit, in der Luft gelassen, wieder sein ursprüngliches Gewicht an.

Für die chemische Analyse wurde ein Stück aus der Mitte des Meteoriten genommen und aus demselben ein gleichartiges Pulver bereitet. Die Analyse begann mit der Untersuchung des im Wasser sich lösenden Teiles.

1 Compt. rend. 109, 976. 
$\mathrm{Zu}$ diesem Zwecke wurden $10 \mathrm{~g}$ des Pulvers mit einem Liter warmen, destillierten Wasser übergossen und in dieser wässerigen Lösung wurde gefunden:

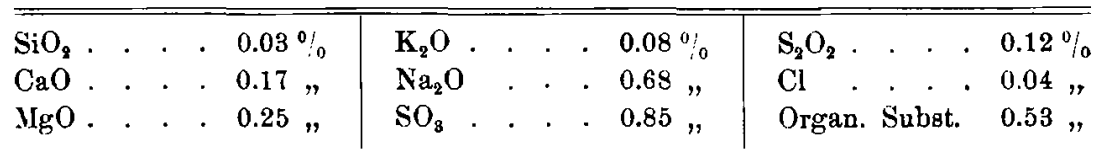

Wie aus dieser Analyse ersichtlich ist, enthält die wässerige Lösung aufser Basen noch Salze der Schwefel- und Thioschwefelsäure; die Anwesenheit letzterer wurde durch charakteristische Reaktionen konstatiert. Schwefelsaure Salze sind wohl durch Oxydation der schwefeligsauren Alkalien und Erdalkalimetalle entstanden. Was aber die thioschwefelsauren Alkalien anbetrifft, so sind sie wahrscheinlich durch Oxydation des Schwefels zu schwefeliger Säure entstanden, welche mit dem freien Schwefel Thioverbindungen bildete. Thioschwefelsaure Salze wurden auch von FrIEDHEIM im Meteorit "Concepcion" gefunden. ${ }^{1}$

Die Natur der im Wasser löslichen organischen Körper des Meteoriten von Migheja war aus folgendem Grunde schwer festzustellen. Ein bekannter Liebhaber und Sammler von Meteoriten, J. Simaschoo, hat den Meteorit, bevor dieser in das mineralogische Kabinett der Neurussischen Universität gebracht wurde, für sich erworben und, wahrscheinlich in der Absicht, einen Abgul's von diesem abzunehmen, hat er ihn mit einer Schicht von Fett bedeckt, welches teilweise auch in das Innere des Meteoriten eingedrungen war. Deshalb haben sich zu den organischen Bestandteilen desselben noch fremde organische Verbindungen beigemischt. Dies erklärt uns die Anwesenheit einiger flüchtigen fetten Säuren, darunter der Ameisensäure, in der wässerigen Lösung.

Es ist sebr zu bedauern, dals so unvorsichtiges Behandeln des Meteoriten die Möglichkeit ausschlofs, die Natur der im Wasser löslichen organischen Körper näher zu untersuchen. Der unlöslich gebliebene Teil des Pulvers wurde mit verdünnter $(30 \%)$ Salzsäure behandelt. Die Auflösung geschah in einer Platinschale, anfangs im kalten, dann während einiger Stunden unter Erwärmen auf dem Wasserbade. Beim Begiefsen des Meteoriten mit Salzsäure machte sich ein schwaches Ansetzen von Schwefelwasserstoff bemerkbar, was auf Anwesenheit von Schwefelmetallen hinweist.

1 Sitzungsberichte der k. Br. Akad. d. Wissenschaften 1888, 363. 
Die Analyse des in $\mathrm{HCl}$ (A) löslichen Teils gab folgende Resultate:

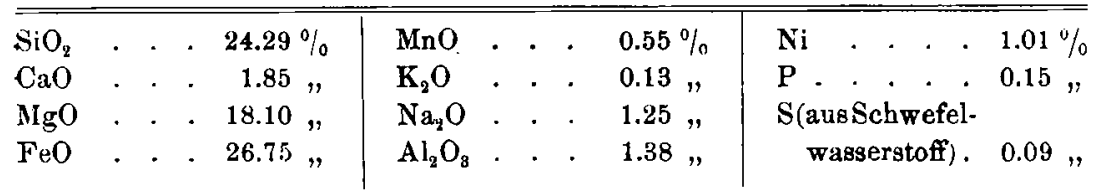

Beim Auszug der Kieselsäure, die sich aus den durch Salzsäure zersetzten Silikaten bildete, durch Soda, wurde zugleich auch Schwefel ausgezogen nebst einem geringen Teil von organischen Stoffen. Darum wurde bei Bearbeitung der Sodalösung mit Salzsäure ein Austreten von Schwefelwasserstoff und eine Ausscheidung von Schwefelmilch beobachtet; aufserdem färbte sich die Flüssigkeit durch die organischen Substanzen etwas braun. Oben ist erwähnt worden, dals der Meteorit thioschwefelsaure Alkalien enthält. Darum durften wir nicht unberücksichtigt lassen, dafs ein Teil des Schwefelwasserstoffes, welcher sich bei Zersetzung der Metallsulfide bildete, durch schwefelige Säure zu Schwefel oxydiert wird. Es mufste daher bei Berechnung der Schwefelmetalle dieser Umstand berücksichtigt werden und zu dem Quantum $0.09 \%$ Schwefel, welches bei der Bestimmung des Schwefelwasserstoffes gefunden wurde, mufste noch $0.08 \%$ Schwefel addiert werden. Diese $0.08 \%$ sind es, welche sich aus dem Schwefelwasserstoff auf Kosten der aus den Thiosäuren sich bildenden schwefeligen Säure oxydieren konnten.

Es mufste somit, den Schwefelmetallen entsprechend, 0.17\% Schwefelwasserstoff vorhanden sein. Das gesamte Quantum Schwefel beträgt $3.69 \%$ und besteht aus folgenden Bestandteilen: Schwefel in Eisensulfid $-0.17 \%$, in Thiosäuren $-0.08 \%$, in Schwefelsäure $-0.34 \%$ und endlich freier Schwefel $-3.10 \%$.

Zur Bestimmung der freien Metalle im Meteorit benutzten wir anfangs das Verfahren von Boussencault, ${ }^{1}$ erhielten aber dabei ungünstige Resultate, weil bei der Behandlung mit Quecksilberchlorid in die Lösung nur ein geringer Teil des Nickels übertrat, welcher durchaus nicht demjenigen Quantum entsprach, welches bei Auflösung des Meteoriten durch Salzsäure gefunden wurde. Was das Eisen anbetrifft, so löste sich keine Spur desselben in Chlorquecksilber. Dieselben Resultate erhielten wir, als an Stelle des Chlor-

1 Etudes s. la transformation du fer en acier, S. 8. 
quecksilbers das Doppelsalz von Chlorquecksilber und Chlorammonium nach dem Verfahren von Friedneim. ${ }^{1}$ angewandt wurde. Eine analoge Erscheinung beobachtete auch FrIEDHEIM bei der Untersuchung des kohlenstoffreichen Meteoriten "Concepcion".

Es nifslang somit die Bestiminung der Menge der freien Metalle auf direktem Wege und wir sahen uns genötigt, die Berechnung indirekt zu vollfübren: nach Verteilung der Kieselsäure unter den Basen (die Thonerde ausgenommen) berechneten wir nach ihrem Reste die entsprechende Menge des Eisenoxyduls für die basischen Silikate; den übrigen Teil des Eisenoxyduls berechneten wir als metallisches Eisen. Der Teil des Metecriten, welcher sich in Salzsäure löste und nach Absonderung der Kieselerde mittels Soda $90.02 \%$ betrug, zeigte folgende Zusammensetzung:

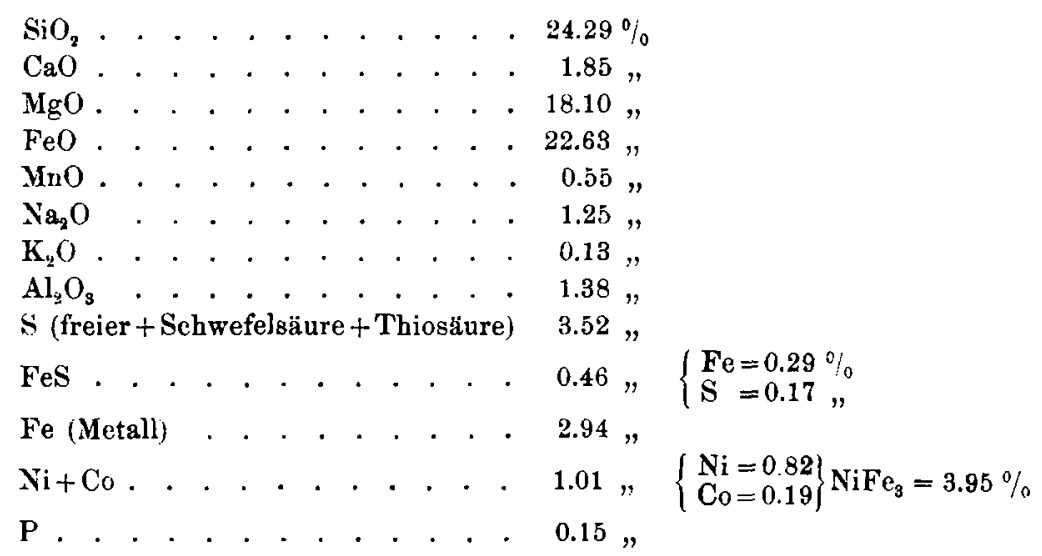

Den in Salzsäure sich lösenden Teil der Silikate aufzählend (die in Wasser sich lösenden Teile abgezogen), und sie für 100 annehmend, erhalten wir folgendes:

$$
\begin{aligned}
& \mathrm{SiO}_{2} \text {. . } 35.91 \% \quad \mathrm{O}=19.15 \% \\
& \mathrm{CaO} \text {. . . } 2.48, \quad \mathrm{O}=0.99 ” \\
& \mathrm{MgO} \text {. . 26.41 " } \mathrm{O}=10.56, \\
& \mathrm{FeO} \cdot \cdot 33.42 " \quad 0=7.44 ",=19.40 \%
\end{aligned}
$$

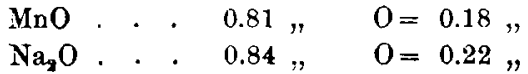

$$
\begin{aligned}
& \mathrm{K}_{2} \mathrm{O} \text {. . . } 0.07, \quad \mathrm{O}=0.01 \text {, }
\end{aligned}
$$

Das Verhältnis des Sauerstoffes der Kieselsäure zum Sauerstoff

1 Etudes s. la transformation du fer en acier, S. 345. 
der Basen zeigt, dafs es ein Mono-Silikat - Olivin - ist von folgender Zusammensetzung:

$$
\begin{aligned}
& 3[\mathrm{MgO}]_{2} \cdot \mathrm{SiO}_{2} \\
& 2[\mathrm{FeO}]_{2} \cdot \mathrm{SiO}_{3},
\end{aligned}
$$

worin ein Teil Magnesium durch Calcium und ein Teil des Eisens durch Mangan ersetzt ist.

Aufserdem enthält der in Säure lösbare Teil (A), wie aus obenangeführter Tabelle ersichtlich, Aluminiumoxyd und Spuren von Chromoxyd.

Der ungelöst gebliebene Teil (B) stellt nach Behandlung mit Salzsäure und Soda eine schwarze Masse vor, welche in Bezug auf den ganzen Meteorit $9.98 \%$ beträgt und aus amorpher Kohle $-2.63 \%$, unlöslichem Silikat $-5.89 \%$ und Chromeisenerz $-1.62 \%$ besteht.

Zur Entfernung der Kohle und zur Untersuchung der mineralischen Bestandteile des unlöslichen Teils des Meteoriten wurde der Rest auf schwachem Feuer geglüht unter starkem Zutritt des Sauerstoffes der Luft, wobei die ganze Kohle rasch verbrannte und eine hellgraue Masse unterblieb. Die mikroskopische Untersuchung dieser Masse zeigte, dafs sie grölstenteils aus rhombischem Pyroxen mit Einschlufs von Glas und geringen Mengen von Chromeisenerz besteht.

Wenn wir das Chromeisenerz aus dem unlöslichen und durchgegluhten Teile ausschliefsen und den Rest zu 100 annehmen, so erhalten wir folgende Zahlen:

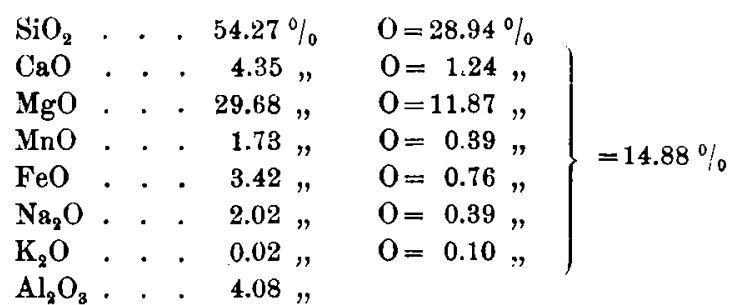

Die chemische Zusammensetzung und das Verhältnis des Sauerstoffs des Silikats zu demjenigen der Basen zeigt, dafs der rhombische Pyroxen ein Enstatit ist. Da wir die Natur der organischen Bestandteile des Meteoriten untersuchen wollten, nahmen wir $20 \mathrm{~g}$ feinen Pulvers und behandelten es mit wasserfreiem Äther bei längerem Erwärmen unter einem Rückflứskühler. Der Äther entzieht die organischen Körper, den Schwefel und einen geringen Teil mineralischer Körper. 
Der Schwefel wurde durch Schütteln der Ätherlösung mit Quecksilber entfernt. Nach Abdestillieren des Äthers blieb ein unbedeutender Rest übrig, welcher aus Fetten bestand, deren Anwesenheit im Meteorit, wie oben erklärt, rein zufällig war; aufserdem aus schweren Kohlenwasserstoffen, welche dem Geruche nach an die schweren Überreste der Naphta erinnerten und weder in Wasser noch in Alkalien sich lösten. Darauf wurden die Kohlenwasserstoffe von den Fetten mittels Verseifung durch eine konzentrierte Ätzkalilösung getrennt. Die erhaltene Menge der Kohlenwasserstoffe war aber eine so geringe, dafs es uns nicht gelang, ihre chemische $\mathrm{Zu}$ sammensetzung näher zu prüfen.

Einige Meteorite, wie z. B. „Concepcion", „Kova", die dem von uns untersuchten analog sind, enthalten stickstoff haltige Substanzen; aus diesem Grunde untersuchten wir den Meteorit von Migheja auch in dieser Hinsicht, erhielten aber negative Resultate.

Die Eigebnisse unserer Untersuchung zusammenstellend, kommen wir $\mathrm{zu}$ folgendem:

1. Der Meteorit besteht aus:

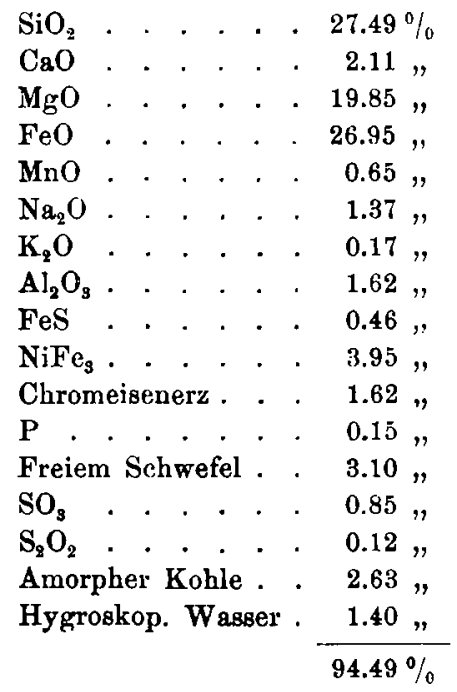

Die übrigen $5.51 \%$ bilden wahrscheinlich organische und andere Körper, welche von uns nicht bestimmt wurden.

2. Der Meteorit von Migheja enthält freien Schwefel, schwefelsaure und thioschwefelsaure Salze und schwefligsaure Metalle. 
3. Die freien Metalle sind nicht ganz in Chlorquecksilber löslich und nur ein Teil des Nickels wird durch dasselbe angegriffen.

4. Das lösliche Silikat besteht hauptsächlich aus Olivin folgender Zusammensetzung:

$$
\begin{aligned}
& 3[\mathrm{MgO}]_{2} \cdot \mathrm{SiO}_{2} \\
& 2[\mathrm{FeO}]_{2} \cdot \mathrm{SiO}_{2}
\end{aligned}
$$

5. Das unlösliche Silikat ist ein Bisilikat aus der Gruppe der Pyroxene - ein Enstatit.

6. Der Meteorit besteht, aufser aus amorpher Kohle, aus Kohlenwasserstoffen, die dem Geruche nach an die schweren Reste der Naphta erinnern.

Odessa, Universität, 1898.

Bei der Redaktion eingegangen am 15. Juli 1898. 